\title{
ON THE GROUP OF AUTOMORPHISMS OF AFFINE ALGEBRAIC GROUPS
}

BY

\author{
DONG HOON LEE
}

\begin{abstract}
We study the conservativeness property of affine algebraic groups over an algebraically closed field of characteristic 0 and of their group of automorphisms. We obtain a certain decomposition of affine algebraic groups, and this, together with the result of Hochschild and Mostow, becomes a major tool in our study of the conservativeness property of the group of automorphisms.
\end{abstract}

1. Introduction. Let $G$ be an affine algebraic group over a field $F$, with Hopf algebra $\mathcal{Q}(G)$ of polynomial functions on $G$, in the sense of [2] and let $W(G)$ denote the group of all affine algebraic group automorphisms of $G$. Then $\mathbb{Q}(G)$ may be viewed as a right $W(G)$-module, with $W(G)$ acting by composition $f \rightarrow f \circ \alpha$ on $\mathcal{Q}(G)$.

We recall, from [3], that $G$ is said to be conservative if $\mathcal{Q}(G)$ is locally finite as a $W(G)$-module. As is shown in [3], the conservativeness of $G$ characterizes the existence of a suitable affine algebraic group structure on $W(G)$ and the obstruction to the conservativeness of a connected $G$ is realized as the presence of certain central tori in $G$, when the base field $F$ is algebraically closed and of characteristic 0 .

In the present study of $W(G)$, we exploit the above results and technique of [3] and, accordingly, we refer to [2] and [3] for standard facts concerning affine algebraic groups and their automorphism group.

The following are brief descriptions of the contents appearing in each section: In \$2, we examine reductive affine algebraic groups and their conservativeness and, in $\$ 3$, we establish a certain $W(G)$-invariant decomposition of $G$ when $G$ is conservative. Finally, in $\S 4$, we use the result obtained in $\$ 3$ to study the structure of $W(G)$.

The following notation is standard throughout: Let $G$ be an affine algebraic group. Then $G_{1}$ denotes the connected component of the identity element of $G$ and $Z(G)$ the center of $G$. If $x \in G$, we use $I_{x}$ to denote the inner automorphism of $G$ that is induced by $x$, and, for a subset $S$ of $G$,

Received by the editors July $9,1976$.

AMS (MOS) subject classifications (1970). Primary 20G15.

Key words and phrases. Affine algebraic groups, conservative, Hopf algebra, antipodes, comultiplication.

- American Mathematical Society 1978 
$\operatorname{Int}_{G}(S)$ denotes $\left\{I_{x}: x \in S\right\}$. In the case where $S=G$, we simply write $\operatorname{Int}(G)$ instead of $\operatorname{Int}_{G}(G)$.

2. Reductive groups and conservativeness. For an affine algebraic group $G$ over a field $F$, let $\mathcal{L}(G)$ denote the Lie algebra of $G$, and for a morphism $\rho$ : $G \rightarrow H$ of affine algebraic groups, $\mathcal{L}(\rho)$ denotes the Lie algebra homomorphism induced by $\rho$. Thus $\mathcal{L}(G)$ consists of all $F$-linear maps $X$ : $\mathcal{Q}(G) \rightarrow F$ such that $X(f g)=X(f) g(1)+f(1) X(g)$ for all $f, g \in \mathcal{L}(G)$, and the map $\mathcal{L}(\rho): \mathcal{L}(G) \rightarrow \mathcal{L}(H)$ is given by $\mathscr{L}(\rho)(X)(f)=X(f \circ \rho), f \in \mathbb{Q}(G)$ and $X \in \mathcal{L}(G)$. For $x \in G$ and $f \in \mathbb{Q}(G)$, we write $x \cdot f$ for the left translate of $f$ by $x$, which is given by $(x \cdot f)(y)=f(y x)$ for $y \in G$ and define $x / f$ : $W(G) \rightarrow F$ by $(x / f)(\alpha)=f(\alpha(x))$.

With this preparation, we prove the following characterization of conservative reductive affine algebraic groups.

THEOREM 2.1. Let $G$ be a reductive affine algebraic group over an algebraically closed field $F$ of characteristic 0 . Then $G$ is conservative if and only if $\operatorname{Int}(G)$ is of finite index in $W(G)$.

Proof. Suppose $\operatorname{Int}(G)$ is of finite index in $W(G)$. Then the Hopf algebra $\mathcal{Q}(G)$ is locally finite as an $\operatorname{Int}(G)$-module. Since $\operatorname{Int}(G)$ is a normal subgroup of $W(G)$, it is then locally finite as a $W(G)$-module, proving that $G$ is conservative.

Suppose, conversely, that $G$ is conservative. Thus, by Theorem 2.1, [4], $W(G)$ is an affine algebraic group and its $F$-algebra $\mathcal{Q}(W(G))$ of polynomial functions on $W(G)$ is generated by the functions $x / f, x \in G$ and $f \in \mathbb{Q}(G)$, and their antipodes.

We first show that the $F$-space $\mathscr{L}(W(G))$ may be identified with an $F$-subspace of the space $Z^{1}(G, \mathcal{L}(G)$ ) of all nonhomogeneous rational 1cocycles of $G$ with coefficients in $\mathcal{L}(G)$ relative to the adjoint action of $G$ on $\mathcal{L}(G)$. To do this, we let $\sigma \in \mathcal{L}(W(G))$ and, for each $x \in G$, we define

$$
\sigma_{x}: \mathcal{Q}(G) \rightarrow F
$$

by

$$
\sigma_{x}(f)=\sigma\left(x / x^{-1} \cdot f\right), \quad f \in \mathbb{Q}(G) .
$$

Then we see easily that $\sigma_{\mathbf{X}} \in \mathcal{L}(G)$ for all $x \in G$, and we also have

$$
\sigma_{x y}=\sigma_{x}+\operatorname{Ad}(x)\left(\sigma_{y}\right), \quad x, y \in G .
$$

To see this, let $\gamma: \mathbb{Q}(G) \rightarrow \mathbb{Q}(G) \otimes \mathbb{Q}(G)$ be the comultiplication of the Hopf algebra $\mathbb{Q}(G)$. For each $f \in \mathbb{Q}(G)$, we write

$$
\gamma(f)=\sum_{i=1}^{n} f_{i} \otimes g_{i}, \quad f_{i}, g_{i} \in \mathscr{Q}(G) .
$$

Then we have 


$$
f(x y)=\sum_{i=1}^{n} f_{i}(x) g_{i}(y) \quad \text { for } x, y \in G .
$$

Now let $\alpha \in W(G)$. Then

$$
\begin{aligned}
\left(x y /(x y)^{-1} \cdot f\right)(\alpha) & =f\left(\alpha(x) \alpha(y) y^{-1} x^{-1}\right) \\
= & f\left(\alpha(x) x^{-1} \cdot I_{x}\left(\alpha(y) y^{-1}\right)\right) \\
= & \sum_{i=1}^{n} f_{i}\left(\alpha(x) x^{-1}\right) g_{i}\left(I_{x}\left(\alpha(y) y^{-1}\right)\right) \quad(\text { by }(3)) \\
= & \sum_{i=1}^{n}\left(x / x^{-1} \cdot f_{i}\right)(\alpha)\left(y / y^{-1} \cdot\left(g_{i} \circ I_{x}\right)\right)(\alpha) .
\end{aligned}
$$

That is, we have

$$
x y /(x y)^{-1} \cdot f=\sum_{i=1}^{n}\left(x / x^{-1} \cdot f_{i}\right) \cdot\left(y / y^{-1}\left(g_{i} \circ I_{x}\right)\right) .
$$

Now

$$
\begin{aligned}
\sigma_{x y}(f) & =\sigma\left(x y /(x y)^{-1} \cdot f\right)=\sigma\left(\sum_{i=1}^{n}\left(x / x^{-1} \cdot f\right) \cdot\left(y / y^{-1}\left(g_{i} \circ I_{x}\right)\right)\right) \\
& =\sum_{i=1}^{n} \sigma\left(x / x^{-1} \cdot f_{i}\right) g_{i}(1)+\sum_{i=1}^{n} f_{i}(1) \sigma\left(y / y^{-1} \cdot\left(g_{i} \circ I_{x}\right)\right) .
\end{aligned}
$$

However, we have (using (3))

$$
\begin{aligned}
x / x^{-1} \cdot f & =\sum_{i=1}^{n}\left(x / x^{-1} \cdot f_{i}\right) g_{i}(1), \text { and } \\
y / y^{-1} \cdot\left(f \circ I_{x}\right) & =\sum_{i=1}^{n}\left(y / y^{-1} \cdot\left(g_{i} \circ I_{x}\right)\right) f_{i}(1)
\end{aligned}
$$

Hence

$$
\begin{aligned}
\sigma_{x y}(f) & =\sigma\left(x / x^{-1} \cdot f\right)+\sigma\left(y / y^{-1} \cdot\left(f \circ I_{x}\right)\right)=\sigma_{x}(f)+\sigma_{y}\left(f \circ I_{x}\right) \\
& =\left(\sigma_{x}+\operatorname{Ad}(x)\left(\sigma_{y}\right)\right)(f),
\end{aligned}
$$

proving (1).

For each $\sigma \in \mathfrak{L}(W(G))$, define $\sigma^{\prime}: G \rightarrow \mathfrak{L}(G)$ by $\sigma^{\prime}(x)=\sigma_{x}, x \in G$. Then we easily see that $\sigma^{\prime} \in Z^{1}(G, \mathcal{L}(G))$. Since the functions $x / f$, together with their antipodes, generate $\mathcal{Q}(W(G))$ as an $F$ :algebra, it follows that the $F$-linear map $\sigma \rightarrow \sigma^{\prime}$ is injective, under which we identify $\mathcal{L}(W(G))$ with an $F$-subspace of $Z^{1}(G, \mathcal{L}(G))$.

We next consider the morphism of affine algebraic groups $\nu: G \rightarrow W(G)$, which is given by $\nu(x)=I_{x}, x \in G$.

We compute the image of $\mathscr{L}(G)$ under the $F$-linear map $\mathscr{L}(v): \mathcal{L}(G) \rightarrow$ 
$\mathcal{L}(W(G)), \mathcal{L}(W(G))$ being identified with an $F$-subspace of $Z^{1}(G, \mathcal{L}(G))$.

To do this, we first note that $X\left(f^{\prime}\right)=-X(f)$ for all $f \in \mathbb{Q}(G)$ and $X \in \mathcal{L}(G)$. This may be seen as follows: Write $\gamma(f)=\sum_{i=1}^{n} f_{i} \otimes g_{i}$ as in (2). Then, by (3),

$$
f(1)=f\left(x x^{-1}\right)=\sum_{i=1}^{n} f_{i}(x) g_{i}^{\prime}(x)=\left(\sum_{i=1}^{n} f_{i} g_{i}^{\prime}\right)(x),
$$

which implies that $\sum_{i=1}^{n} f_{i} g_{i}^{\prime}$ is constant.

Hence

$$
\begin{aligned}
0 & =X\left(\sum_{i=1}^{n} f_{i} g_{i}^{\prime}\right)=\sum_{i=1}^{n} X\left(f_{i}\right) g_{i}^{\prime}(1)+\sum_{i=1}^{n} f_{i}(1) X\left(g_{i}^{\prime}\right) \\
& =X\left(\sum_{i=1}^{n} f_{i} g_{i}(1)\right)+X\left(\sum f_{i}^{\prime}(1) g_{i}^{\prime}\right) \\
& =X(f)+X\left(f^{\prime}\right)
\end{aligned}
$$

and $X\left(f^{\prime}\right)=-X(f)$ follows.

For $X \in \mathcal{L}(G), x \in G$, and $f \in \mathbb{Q}(G)$, we have

$$
\mathfrak{L}(\nu)(X)(x)(f)=\mathfrak{L}(\nu)(X)\left(x / x^{-1} \cdot f\right)=X\left(\left(x / x^{-1} f\right) \cdot \nu\right) .
$$

But $\left(x / x^{-1} \cdot f\right) \cdot \nu=\sum_{i=1}^{n} f_{i} \cdot\left(g_{i} \cdot \nu(x)\right)^{\prime}$.

Hence

$$
\begin{aligned}
\mathcal{L}(\nu)(X)(x)(f) & =X\left(\sum_{i=1}^{n} f_{i} \cdot\left(g_{i} \cdot \nu(x)\right)^{\prime}\right) \\
& =\sum_{i=1}^{n} X\left(f_{i}\right)\left(g_{i} \cdot \nu(x)\right)^{\prime}(1)+\sum_{i=1}^{n} f_{i}(1) X\left(g_{i} \cdot \nu(x)^{\prime}\right) \\
& =X\left(\sum_{i=1}^{n} f_{i} g_{i}(1)\right)-X\left(\sum_{i=1}^{n} f_{i}(1)\left(g_{i} \cdot \nu(x)\right)\right) \\
& =X(f)-X(f \cdot \nu(x))=(X-\operatorname{Ad}(x)(X))(f) .
\end{aligned}
$$

That is, $\mathscr{L}(\nu)(X)(x)=X-\operatorname{Ad}(x)(X)$, and we see that $\operatorname{Im}(\mathfrak{L}(\nu))$ is equal to the subsapce $B^{1}(G, \mathfrak{L}(G))$ of $Z^{1}(G, \mathcal{L}(G))$ consisting of all 1-coboundaries of G.

Since $G$ is reductive, $H^{1}(G, \mathcal{L}(G))=0$. Hence $\operatorname{Im}(\mathscr{L}(\nu))=B^{1}(G, \mathscr{L}(G))=$ $Z^{1}(G, \mathcal{L}(G))$. Since $F$ is algebraically closed, the surjectivity of $\mathfrak{L}(\nu)$ implies that $\operatorname{Im}(\nu)=\operatorname{Int}(G)$ is open in $W(G)$ and hence $\operatorname{Int}(G)$ is of finite index in $W(G)$.

THEOREM 3.2. Let $G$ be an affine algebraic group over an algebraically closed 
field $F$ of characteristic 0 . Then $G$ is conservative if a maximal reductive subgroup of $G$ is conservative.

Proof. Let $G_{u}$ denote the unipotent radical of $G$, and let $P$ be a maximal reductive subgroup of $G$. Since $F$ is of characteristic 0 , a theorem of Mostow (see [2, Theorem 14.2]) assures that we have a semidirect product decomposition $G=G_{u} \cdot P$. By the conjugacy of maximal reductive subgroups, we may assume that $P$ is conservative, and we have $W(G)=\operatorname{Int}(G) \cdot \mathscr{A}$, where $\mathscr{A}$ is the subgroup of $W(G)$ consisting of all $\alpha \in W(G)$ leaving $P$ invariant.

Let $\mathscr{A}_{P}$ denote the restriction image of $\mathscr{A}$ in $W(P)$. Then $\operatorname{Int}(P)<\mathscr{A}_{p}$, and, since $P$ is conservative, $W(P) / \operatorname{Int}(P)$ is finite by Theorem 2.1. It follows that $\mathscr{A}_{p} / \operatorname{Int}(P)$ is also finite.

From this point on, we can copy the argument used in [3, p. 539] for the proof of conservativeness of $G$ when $P$ is a connected semi-simple algebraic subgroup and conclude that $G$ is conservative. This establishes Theorem 2.2.

3. $W(G)$-invariant decomposition of $G$. For a subset $\mathscr{A}$ of $W(G)$, let $G^{\mathscr{A}}$ denote the set consisting of all $x \in G$ such that $\alpha(x)=x$ for all $\alpha \in \mathscr{A}$.

We prove the following result which will then be used in $\$ 4$ for out study of $W(G)$.

THEOREM 3.1. Let $G$ be a connected conservative affine algebraic group over an algebraically closed field $F$ of characteristic 0 , and let $T$ be the maximal central torus of $W(G)_{1}$. Then there exists a $W(G)$-invariant algebraic vector subgroup $Z$ of $G$ such that $G=Z \times G^{T}$.

Proof. If $T$ is trivial, then the assertion holds trivially. Thus we assume that $T$ is of dimension $\geqslant 1$.

For each $x \in G$, the inner automorphism $I_{x}$ induced by $x$ commutes with every element of $T$. Hence, for $\alpha \in T$ and $x \in G$, we have $x^{-1} \alpha(x) \in Z(G)$.

We define, for each $\alpha \in T, \eta_{\alpha}: G \rightarrow Z(G)$ by $\eta_{\alpha}(x)=x^{-1} \alpha(x), x \in G$.

Then $\eta_{\alpha}$ is a morphism of affine algebraic groups. Since $G$ is connected, it follows that $\eta_{\alpha}(x) \in Z(G)_{1}$ for all $x \in G$. Now we choose a maximal reductive subgroup $P$ of $G$ so that $G=G_{u} \cdot P$ (semidirect). We first show that every element of $P$ is $T$-fixed. To do this, we choose a maximal torus $D$ of $P$. Then $P=D \cdot P^{\prime}$, where $P^{\prime}$ denotes the commutator subgroup of $P$, and $P^{\prime}<\operatorname{Ker} \eta_{\alpha}$ implies that every element of $P^{\prime}$ is $T$-fixed. Hence it is enough to show that every element of $D$ is $T$-fixed. Let $K$ be the maximal torus of $Z(G)$. Then the torus $\eta_{\alpha}(D)$ is contained in $K$, and hence we see that every element $\alpha$ of $T$ leaves $D$ invariant. Consider the polynomial map

$$
\phi: T \times D \rightarrow D \text {, }
$$

given by $\phi(\alpha, x)=\alpha(x)$, and define, for each $x \in D, \phi_{x}: T \rightarrow D$ by $\phi_{x}(\alpha)=$ $\alpha(x)$. Then clearly $\phi_{x}$ is a polynomial map. Let $x \in D$ be of order $m<\infty$. 
Then $\phi_{x}(\alpha)$ is also of order $m$ for all $\alpha \in T$. Since $D$ contains only a finite number of elements of order $m$, it follows from the connectedness of $T$ that Im $\phi_{x}=\{x\}$. That is, $\alpha(x)=x$ for all $\alpha \in T$. Since the elements in $D$ of finite order form a dense subset of $D$, it follows that $T$ leaves every element of $D$ fixed.

Next we show that if $U$ denotes the unipotent radical of $Z(G)$, then $G=U \cdot G^{T}$. The morphism $\eta_{\alpha}: G \rightarrow Z(G)$ for $\alpha \in T$ maps $G_{u}$ into $U$. Hence $\eta_{\alpha}$ induces a morphism $\mu_{\alpha}: G_{u} \rightarrow U$ of affine algebraic groups. Let $\mu_{\alpha}^{0}$ denote $\mathcal{L}\left(\mu_{\alpha}\right): \mathcal{L}\left(G_{u}\right) \rightarrow \mathfrak{L}(U)$. The natural action of $T$ on $U$ determines a $T$-module structure on the $F$-space $\mathcal{L}(U)$, and this in turn defines a $T$-module structure on the $F$-space $\operatorname{Hom}_{F}\left(\mathcal{L}\left(G_{u}\right), \mathcal{L}(U)\right)$.

We then have

$$
\mu_{\beta}^{0}=\mu_{\beta}^{0}+\alpha \cdot \mu_{\beta}^{0}, \quad \alpha, \beta \in T .
$$

To prove (1), we note that $\exp _{U} \cdot \mu_{\alpha}^{0}=\mu_{\alpha} \cdot \exp _{G_{u}}$, where $\exp _{U}$, $\exp _{G_{u}}$ denote the exponential maps for $U, G_{u}$, respectively. Hence for $X \in \mathcal{L}\left(G_{u}\right)$,

$$
\begin{aligned}
\exp \mu_{\alpha \beta}^{0}(X) & =\mu_{\alpha \beta}(\exp X)=(\exp X)^{-1} \alpha \beta(\exp X) \\
= & (\exp X)^{-1} \alpha(\exp X) \alpha\left((\exp X)^{-1} \beta(\exp X)\right) \\
= & \mu_{\alpha}(\exp X) \alpha\left(\mu_{\beta}(\exp X)\right)=\exp \mu_{\alpha}^{0}(X) \alpha\left(\exp \mu_{\beta}^{0}(X)\right) \\
= & \exp \mu_{\alpha}^{0}(X) \exp \left(\mathcal{L}(\alpha)\left(\mu_{\beta}^{0}(X)\right)\right)=\exp \left(\mu_{\alpha}^{0}(X)+\alpha \cdot \mu_{\beta}^{0}(X)\right) .
\end{aligned}
$$

Hence it follows that $\mu_{\alpha \beta}^{0}(X)=\mu_{\alpha}^{0}(X)+\alpha \cdot \mu_{\beta}^{0}(X)$, proving (1).

The identity (1) defines a rational $T$-module structure on the $F$-space $F \oplus \operatorname{Hom}_{F}\left(\mathcal{L}\left(G_{u}\right), U\right)$, if we define the $T$-action by $\alpha \cdot(r, \phi)=\left(r, r \mu_{\alpha}^{0}+\alpha\right.$. $\phi)$ for $\alpha \in T, r \in F$ and $\phi \in \operatorname{Hom}_{F}\left(\mathscr{L}\left(G_{u}\right), \mathcal{L}(U)\right)$. Since $T$ is reductive, the $T$-submodule $\operatorname{Hom}_{F}\left(\mathcal{L}\left(G_{u}\right), \mathcal{L}(U)\right)$ has a 1-dimensional $T$-invariant complement in $F \oplus \operatorname{Hom}_{F}\left(\mathcal{L}\left(G_{u}\right), \mathcal{L}(U)\right)$. This complement contains exactly one element of the form $(1, \phi)$.

Hence $(1, \phi)=\alpha \cdot(1, \phi)=\left(1, \mu_{\alpha}^{0}+\alpha \cdot \phi\right)$ for all $\alpha \in T$ and this implies that $\mu_{\alpha}^{0}=\phi-\alpha \cdot \phi, \alpha \in T$.

For each $X \in \mathcal{L}\left(G_{u}\right)$, we have

$$
\begin{aligned}
\exp \phi(X) & =\exp \left(\mu_{\alpha}^{0}(X)+\alpha \cdot \phi(X)\right) \\
& =\exp \left(\mu_{\alpha}^{0}(X)\right) \exp (\mathcal{L}(\alpha)(\phi(X))) \\
& =(\exp X)^{-1} \alpha(\exp X) \alpha(\exp \phi(X)) .
\end{aligned}
$$

Hence $\exp _{G_{u}} X \cdot \exp _{U} \phi(X) \in G^{T}$ for all $X \in \mathcal{L}\left(G_{u}\right)$. Since $\exp _{G_{u}}\left(\mathcal{L}\left(G_{u}\right)\right)=$ $G_{u}$, it follows that $G_{u}<U \cdot G^{T}$, and $p<G^{T}$ implies $G=U \cdot G^{T}$.

Now we consider the rational $T$-module $\mathcal{L}(U)$. Since $T$ is a torus over an algebraically closed field, we may decompose the $F$-space $\mathcal{L}(U)$ as 


$$
\mathcal{L}(U)=\sum_{x \neq 1} L_{x}+\mathcal{L}(U)^{T},
$$

where $L_{\chi}$ is the weight space $\{X \in \mathcal{L}(U): \alpha \cdot X=\chi(\alpha) X$ for all $\alpha \in T\}$ corresponding to the weight $\chi: T \rightarrow F^{*}$, and $\mathscr{L}(U)^{T}$ is the $T$-fixed part of $\mathcal{L}(U)$.

Since $T$ is a normal subgroup of $W(G), W(G)$ permutes the weights of $T$ in $\mathcal{L}(U)$. Hence the $F$-subspace $Z=\sum_{\chi=1} L_{\chi}$ is $W(G)$-invariant. Let $Z=$ $\exp _{U} Z$. Then $U=Z \times U^{T}$ and this implies that $G=Z \times G^{T}$ follows. Clearly $Z$ is $W(G)$-invariant and the theorem is proved.

REMARK. Since $T$ is a normal subgroup of $W(G)$, it follows that $G^{T}$ is also $W(G)$-invariant. As we will see in $\S 4, T$ is central in $W(G)$ and, in fact, a direct factor of $W(G)$.

\section{Decomposition and conservativeness of $W(G)$.}

THEOREM 4.1. Let $G$ be a conservative connected affine algebraic group over an algebraically closed field $F$ of characteristic 0 . Then the maximal central torus of $W(G)_{1}$ is of dimension $\leqslant 1$ and is a direct factor of $W(G)$.

Proof. Let $T$ be the maximal central torus of $W(G)_{1}$, and assume that $T$ is nontrivial. Then we have a $W(G)$-invariant decomposition $G=Z \times G^{T}$ (Theorem 3.1). Hence we have $W(G) \simeq W(Z) \times W\left(G^{T}\right)$ as affine algebraic groups and the restriction map $T \rightarrow W(Z)$ is injective.

Let $z$ denote the Lie algebra of $Z$. Then the affine algebraic group $W(Z)$ may be identified with the affine algebraic group $G L(z)$ of all $F$-linear automorphisms of $z$. Since $F$ is algebraically closed, the center of $W(Z)$ is a 1-dimensional torus and is a direct factor of $W(Z)$. Since every element of $W(Z)$ can be extended to an element of $W(G)$, we see easily that the restriction map sends $T$ isomorphically onto the center of $W(Z)$. Hence our assertion follows.

In [2], Hochschild proved that, if $G$ is a nonabelian unipotent affine algebraic group, then the maximal central torus of $W(G)_{1}$ is trivial and hence that $W(G)_{1}$ is conservative. The assertion does not hold for arbitrary solvable affine algebraic groups (see the example in [2, p. 111]).

The following theorem characterizes those nonabelian solvable groups $G$ for which $W(G)_{1}$ is conservative.

THEOREM 4.2. Let $G$ be a connected conservative solvable nonabelian affine algebraic group over an algebraically closed field of characteristic 0 . Then the following are equivalent:

(i) $W(G)_{1}$ is conservative.

(ii) The connected component of the center of $W(G)_{1}$ is unipotent (i.e. $T=1)$. 
(iii) $G$ cannot be a product $G=Z \times H$ of a nontritial algebraic vector subgroup $Z$ and an algebraic subgroup $H$, both of which are invariant under $W(G)$.

Proof. (iii) $\rightarrow$ (ii) follows from Theorem 3.1 and the subsequent remark.

(ii) $\rightarrow$ (iii) holds because of the decomposition $W(G)=W(Z) \times W(H)$.

(ii) $\rightarrow$ (i) follows from Theorem 3.2 of [4].

It remains to show (i) $\rightarrow$ (ii).

Let $K$ be a maximal torus of $G$ so that $G=G_{u} \cdot K$ (semidirect).

If $K$ is trivial, then $G$ is unipotent and nonabelian, and hence (ii) holds (see [2, p. 110]).

(1) Suppose $\operatorname{dim} K \geqslant 2$. Then the maximal central torus of $G$ is trivial by Theorem 3.2 [4] and this implies that the torus $\operatorname{Int}_{G}(K) \simeq K Z(G) / Z(G)$ is of dimension $\geqslant 1$. Since $\operatorname{Int}(G)$ is a normal algebraic subgroup of $W(G)$, it follows that the algebraic torus $\operatorname{Int}_{G}(K)$ is contained in the radical of $W(G)_{1}$ and hence is central in a maximal reductive group containing it. Since $W(G)_{1}$ is conservative, (ii) follows from Theorem 3.2 of [3].

(2) Suppose $\operatorname{dim} K=1$. If $K$ is central in $G$, then $G=G_{u} \times K$, and hence $W(G) \simeq W\left(G_{u}\right) \times \mathbf{Z}_{2}$. Since $G_{u}$ is nonabelian, (ii) follows immediately.

Therefore we may assume that the identity component of the center of $G$ is unipotent. Then $\operatorname{Int}_{G}(K)$ is a 1-dimensional torus. Assume that (ii) does not hold, and let $T$ be the maximal central torus of $W(G)_{1}$. Then $T \cap \operatorname{Int}_{G}(K)=$ $\{1\}$, for if $\alpha \in T$ is of the form $\alpha=I_{x}$ for some $x \in K$, then the decomposition $G=Z \times G^{T}$ in Theorem 3.1 implies that $\alpha=1$.

Since $T$ centralizes $\operatorname{Int}_{G}(K)$, it follows that $T^{\prime}=T \cdot \operatorname{Int}_{G}(K)$ (direct) is an algebraic torus of dimension 2 .

Since $T^{\prime}$ is contained in the radical of $W(G)_{1}$, it follows that $T^{\prime}$ is central in a maximal reductive subgroup containing $T^{\prime}$. (See [1, Chapter III].) Hence again by Theorem 3.2 of [3], $W(G)$, cannot be conservative, contradicting (i). Therefore $T=\{1\}$ and (ii) is proved.

\section{REFERENCES}

1. A. Borel, Linear algebraic groups, Benjamin, New York, 1969. MR 40 \#273.

2. G. Hochschild, Introduction to affine algebraic groups, Holden-Day, San Francisco, 1971. MR 43 \#3268.

3. G. Hochschild and G. D. Mostow, Automorphisms of affine algebraic groups, J. Algebra 22 (1972), 535-543.

Department of Mathematics, Case Western Reserve University, Cleveland, Oho 44106 